\title{
Acute Epiglottitis Due to COVID-19 Infection
}

\author{
Jonathan Emberey ${ }^{1}$, S Srinath Velala², Ben Marshall ${ }^{3}$, Adil Hassan ${ }^{1}$, Salim PL Meghjee ${ }^{1}$, M Jamil Malik ${ }^{1}$, Muhammad Hussain ${ }^{1}$ \\ ${ }^{1}$ Department of Respiratory Medicine, Barnsley Hospital NHS Foundation Trust, Barnsley, UK \\ ${ }^{2}$ Department of ENT/Otolaryngology - Head \& Neck Surgery, Barnsley Hospital NHS Foundation Trust, Barnsley, UK \\ ${ }^{3}$ Department of Anaesthetics, Barnsley Hospital NHS Foundation Trust, Barnsley, UK
}

\section{Doi: 10.12890/2021_002280 - European Journal of Case Reports in Internal Medicine - @ EFIM 2021}

Received: 10/01/2020

Accepted: $11 / 01 / 2021$

Published: 03/03/2021

\begin{abstract}
How to cite this article: Emberey J, Velala SS, Marshal B, Hassan A, Meghjee SPL, Malik MJ, Hussain M. Acute epiglottitis due to COVID-19 infection.
\end{abstract} EJCRIM 2021;8: doi:10.12890/2020_002280.

Conflicts of Interests: The Authors declare that there are no competing interests.

This article is licensed under a Commons Attribution Non-Commercial 4.0 License

\section{ABSTRACT}

A 53-year-old man presented acutely to the Accident and Emergency department with a 2-day history of progressive odynophagia and shortness of breath. The patient had stridor at rest and acute epiglottitis was suspected. The patient was transferred urgently to theatre for intubation but due to a severely oedematous airway, this was unsuccessful and emergency tracheotomy was performed by the ENT team. Throughout admission the only positive microbiological sample was a nasopharyngeal swab for SARS-CoV-2 infection. In the absence of other positive microbiology, it is highly likely that COVID-19 was the aetiological cause of acute epiglottitis in this instance.

\section{LEARNING POINTS}

- COVID-19 infection is a novel disease with multiple presentations; it should be considered as a possible causative organism in patients presenting with acute epiglottitis.

- Due to the time delay in taking samples for microbiology and results being available, treatment should be commenced with antibiotics, nebulised adrenaline and steroids to cover bacterial infection.

- Presentation can occur following a delayed inflammatory response and treatment should target the organ system involved.

\section{KEYWORDS}

Epiglottitis, COVID-19

\section{CASE DESCRIPTION}

A 53-year-old male non-smoker, with no medical comorbidities or drug allergies, presented acutely to the Accident and Emergency department with a 2-day history of progressive odynophagia, neck swelling and shortness of breath. The patient had a hoarse voice, trismus and stridor at rest. He was alert and oxygen saturations were $91 \%$ on room air, with a clear chest on auscultation. Treatment was commenced with nebulised adrenaline, intravenous hydrocortisone and benzylpenicillin, and both ENT and ITU teams were alerted to a potential airway emergency.

Examination by the ENT team revealed a tender anterior neck, grade 3 non-inflamed tonsils and on nasoendoscopy a swollen, erythematous and non-purulent epiglottis, with inability to visualise the vocal cords. The patient was transferred to theatre and anaesthetised for intubation by the ITU team. Intubation was unsuccessful due to extensive airway swelling and an emergency tracheotomy was performed. The patient was successfully ventilated using bag and mask maintaining saturations of $95 \%$ throughout. 
The patient was admitted to ITU for 3 days and treated with intravenous ceftriaxone $1 \mathrm{~g}$ twice a day and intravenous dexamethasone $6.6 \mathrm{mg}$ three times a day on the advice of the ENT team. He was stepped down to respiratory HDU and continued to do well on the ward. Followup endoscopy was performed on day 5, showing ongoing supraglottic and epiglottic oedema, and on day 8 showing a significantly improved upper respiratory tract (Fig. 1). A chest x-ray showed clear lung fields (Fig. 2). The tracheotomy was decannulated on day 13, and the patient discharged to be followed up routinely by the ENT team.

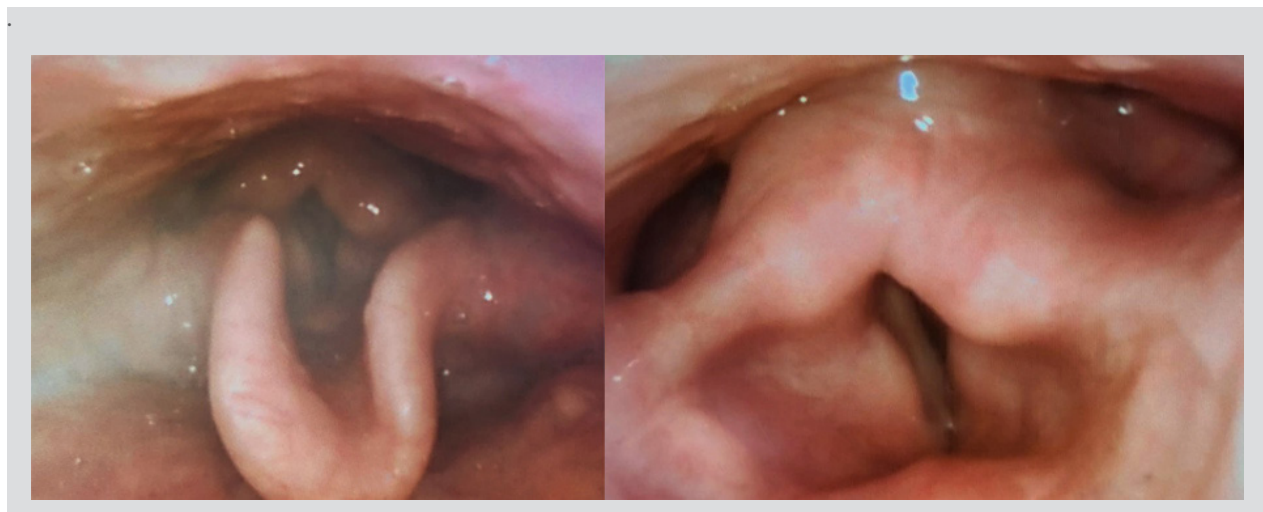

Figure 1. Resolving epiglottic oedema and arytenoid cartilage on day 8 after acute upper airway obstruction

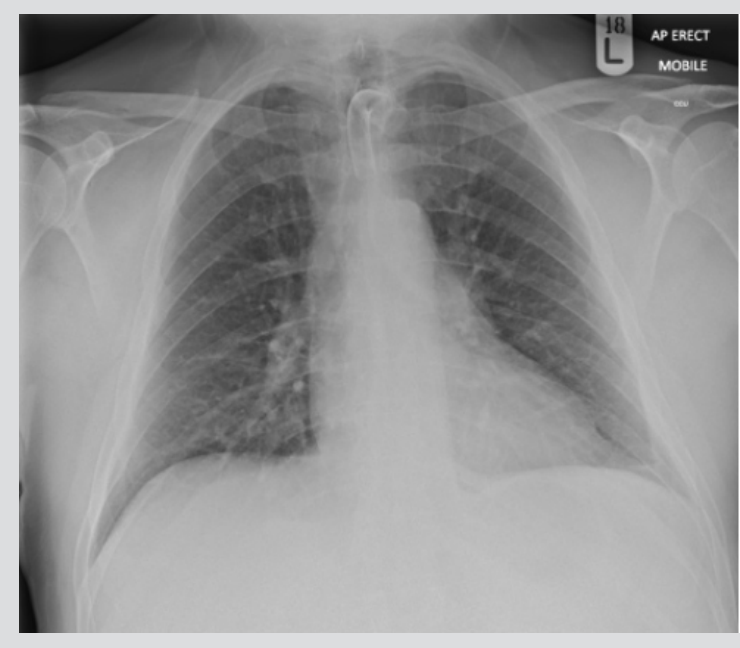

Figure 2. Chest $x$-ray performed 5 days after emergency tracheotomy, showing tracheostomy tube and clear lung fields

On admission, nasopharyngeal swabs demonstrated evidence of SARS-CoV-2 infection, with no evidence of any other respiratory viruses or bacterial organism. This was the patient's second positive COVID-19 test, as he had tested positive in the community 3 weeks earlier. Initial blood film showed leucocytosis $\left(15.7 \times 10^{9}\right.$ cells per litre) with neutrophilia and an initially normal lymphocyte count, falling to lymphopenic levels of $0.7 \times 10^{9}$ cells per litre on day 3 of admission. CRP was elevated $(95 \mathrm{mg} / \mathrm{l})$ and procalcitonin was low $(0.12 \mathrm{ng} / \mathrm{ml})$, suggesting bacterial infection was unlikely. Blood cultures were negative at 48 and 120 hours. Two separate sputum cultures grew no bacteria. COVID-19 blood panels were taken, with D-dimer levels mildly elevated at $0.7 \mu \mathrm{g} / \mathrm{ml}$ (normal range $<0.5)$, troponin I low $(7 \mathrm{ng} / \mathrm{l})$, ferritin normal $(271 \mu \mathrm{g} / \mathrm{l})$ and LDH mildly elevated (303 U/I).

\section{DISCUSSION}

It is well recognised that COVID-19 infection can present with upper airway symptoms, most commonly pharyngodynia, nasal congestion, rhinorrhoea and olfactory dysfunction ${ }^{[1]}$. Here we present a rare new manifestation of the virus, acute epiglottitis with life-threatening upper airway obstruction.

Epiglottitis is an acute inflammation of the epiglottis and surrounding supraglottic structures of the arytenoids and aryepiglottic folds ${ }^{[2]}$. It is typically bacterial in aetiology ${ }^{[2,3]}$, however it may also be precipitated by viral or fungal infection, trauma and smoking. Historically, haemophilus influenza (Hib) was the most common cause of epiglottitis ${ }^{[2,4]}$, but since the introduction of childhood vaccination, the incidence is decreasing in children and increasing in adults ${ }^{[2-4]}$. 
In adults, the typical presentation is over several days ${ }^{[2,3]}$, and the most common symptoms reported are sore throat, odynophagia, fever, shortness of breath, hoarseness, muffled voice, drooling and stridor ${ }^{[2,3]}$. Epiglottitis can rapidly progress to life-threatening airway obstruction ${ }^{[4]}$, and when suspected, arrangements should be made to secure the airway urgently ${ }^{[2]}$. Supportive treatment with humidified oxygen, nebulised adrenaline, empiric antibiotic therapy to cover suspected organisms ${ }^{[2]}$, and intravenous steroid should be given when the airway is secure.

It is often difficult to establish the causative organism in acute epiglottitis. Only $10 \%$ of cases become bacteraemic ${ }^{[2]}$, and in many cases viral agents are suspected when no other organism is isolated ${ }^{[3]}$. In this case, the initial blood panel demonstrating leucocytosis and lymphopenia was suggestive of COVID-19 infection, and active infection with SARS-CoV-2 was confirmed twice with positive nasopharyngeal swabs. Bacterial throat swab, sputum culture, blood culture, and further investigation for respiratory viruses were all negative. Appearances at nasoendoscopy were non-purulent, again suggestive of a non-bacterial cause. Evidence suggests that infection with COVID-19 virus can lead to excessive upregulation of the host inflammatory response through repeat epithelial and endothelial damage leading to a cytokine storm ${ }^{[5]}$. We propose the delayed, severe epiglottic inflammation seen here (after initial infection with COVID-19) could be a result of this mechanism.

A review of literature via PubMed found only one case of epiglottitis with concomitant COVID-19 infection ${ }^{[4]}$. In this case, a 60-year-old man presented with acute upper airway obstruction requiring emergency cricothyroidotomy. The authors proposed e-cigarette use as the most likely cause, but also theorised that COVID-19 could be a potential infectious cause ${ }^{[4]}$ as all other microbiological tests were negative. COVID-19 infection is well documented to cause upper respiratory tract symptoms, and as such we believe that in the absence of any other positive microbiological investigations, it is highly likely that COVID-19 was the aetiological cause of acute epiglottitis in this instance.

\section{REFERENCES}

1. Lovato A, de Filippis C. Clinical presentation of COVID-19: a systematic review focusing on upper airway symptoms. Ear Nose Throat J $2020 ; 99(9): 569-576$.

2. Durand M, Deschler D. Epiglottitis, acute laryngitis, and croup. Infections of the Ears, Nose, Throat, and Sinuses 2018;4:247-255.

3. Orhan I, Aydin S, Karlidag T. Infectious and noninfectious causes of epiglottitis in adults, review of 24 patients. Turk Arch Otorhinolaryngol 2015;53(1):10-14.

4. Fondaw A, Arshad M, Batool S, Robinson B, Patel T. COVID-19 infection presenting with acute epiglottitis. J Surg Case Rep 2020;2020(9):rjaa280.

5. Yanuck S, Pizzorno J, Messier H, Fitzgerald K. Evidence supporting a phased immuno-physiological approach to COVID-19 from prevention through recovery. ICMJ 2020;19(Suppl 1):8-35. 